\title{
Heterodyn-Speckle-Interferometrie zur Schwingungsmessung
}

G. Jahn, H.J. Tiziani

Institut für Technische Optik, Universität Stuttgart

Pfaffenwaldring 9, D 7000 Stuttgart 80

Einleftung

Die gebräuchlichsten Messverfahren auf dem Gebiet der optischen Schwingungs- und Verformungsmesstechnik sind die holographische Interferometrie, die (elektronische) Speckle-Interferometrie und die LaserDoppler-Velocimetrie /1/./2/. Mit den beiden erstgenannten Verfahren können Schwingungsamplituden oder Verformungen an Flächen gemessen werden, ait dem letztgenannten Verfahren der zeitliche Verlauf der Geschwindigkeit an einem Punkt des Messobjekts. Eine Kombination aus Speckle-Interferometrie und Doppler-Velocimetrie ermöglicht die Messung von Schwingungen bzw. Geschwindigkeiten an rauhen oberflächen an mehreren Punkten gleichzeitig. Wir nennen dieses Verfahren Heterodyn-Speckle-Interferometrie.

Das Messprinzip ist das der Laser-Speckle-Interferometrie, die durch Einführung einer Lichtfrequenzverschiebung im Referenzstrahl dynamisiert wird. Dem Speckle-Bild des Messobjekts wird die Referenzwelle überlagert, gemessen wird die Frequenz der Intensitatsanderung an bestimmten Punkten des Bildes. Diese Frequenz $f(t)$ ist die Summe aus der konstanten Frequenzverschiebung $f$ in Referenzstrahl und der Dopplerverschiebung des Lichts aus det zugehörigen bewegten oberflächenelement. Die Geschwindigkeit ist dann $v(t)=f_{d}(t) \cdot \lambda / 2=$ $\left(f(t)-f_{r}\right) \cdot \lambda / 2$, man erhält die Geschwindigkeftskomponente in Beobachtungsrichtung an den stellen des objekts, an denen im Bild die Frequenz gemessen wird. Die Verformung ergibt sich durch Integration der Geschwindigke1t, ausgehend vom Zustand bef Beginn der Frequenzmessung.

\section{Messaufbau}

Der Aufbau des Heterodyn-Speckle-Interferometers ist in Abb. 1 schematisch dargestellt. Das Licht eines 15 wh He-Ne-Lasers wird durch ein rotierendes Radialphasengitter /3/,/4/ in Beleuchtungs- und Refe- 


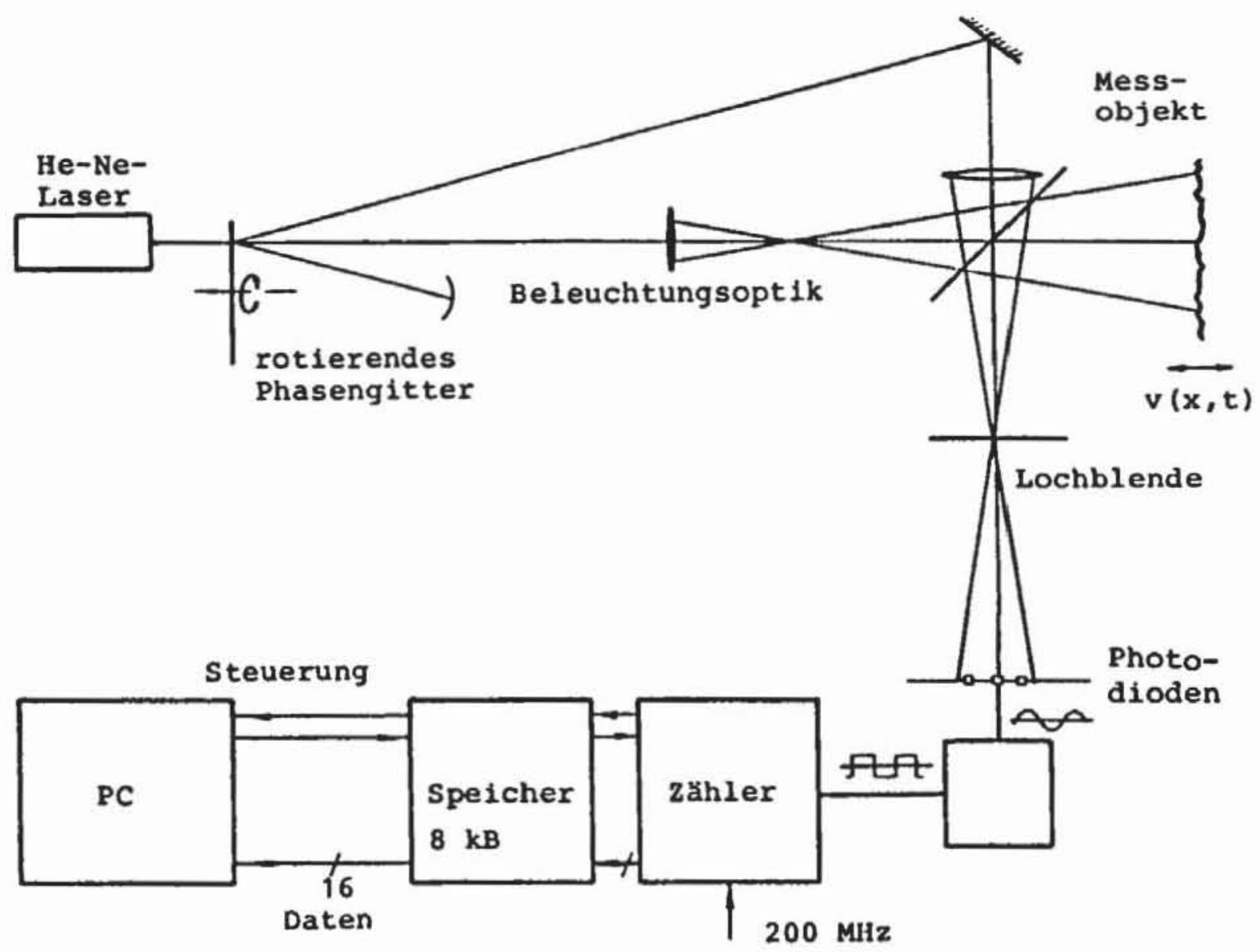

Abb. 1. Schematischer Aufbau des Heterodyn-Speckle-Interferometers

renzstrahl geteilt. Die Rotation des Gitters hat im gebeugten Licht eine Frequenzverschiebung zur Folge, deren Grösse gegeben ist durch Liniendichte des Gitters $x$ Umfangsgeschwindigke1t $x$ Beugungsordnung. Das verwendete Gitter ermöglicht eine Frequenzverschiebung $z w i s c h e n ~ 80$ $\mathrm{kHz}$ und $4 \mathrm{MHz}$, der Geschwindigkeitsmessbereich ist somit $+/-1.44$ $\mathrm{m} / \mathrm{sec}$. Das ungebeugte Licht beleuchtet das Messobjekt, das mit einer Lochkamera abgebildet wird. In der Bildebene sind an den interessierenden Stellen PIN-Photodioden angebracht llichtempfindiche Flăche $\approx 1 \mathrm{~mm}^{2}$ ).

Es ist ein sehr kleines offnungverhältnis nötig, damit die Speckles so gross werden, dass die alfttlere Specklegrösse etwa gleich der Diodenfläche 1st. Lăgen mehrere Speckles in der Diodenfläche, so würde sich durch Mittelung das Nutzsignal verkleinern. Unter diesen Gegebenheiten erweist sich eine Lochkamera als ausreichend, weitere Abbildungsoptik ist nicht erforderlich.

Die Referenzwelle wird auf die Lochblende fokussiert, sie überdeckt 
das ganze Bild des Objekts in der Ebene, in der sich die Photodioden befinden. Die Ausdehnung der Messfläche hängt von der zur Verfügung stehenden Laserleistung ab. Ein Laser mit $15 \mathrm{~mW}$ reicht für flächenbeleuchtung nicht aus ( bei Abstand Interferometer - Messobjekt etwa $20 \mathrm{~cm}$, Objekt mit Reflexfolie 1, möglich ist linienförmige Beleuchtung (etwa $5 \mathrm{~mW} / \mathrm{cm}$ ) oder Aufspaltung des Strahls und Punktbeleuchtung nur der Messpunkte ( etwa $7 \mathrm{~mW}$ pro Punkt).

Zur Signalauswertung, d.h. Frequenzmessung, dient ein $2 a ̈ h l p r o z e s s o r$. Das Photodiodensignal wird verstärkt, bandpassgefiltert und in ein Rechtecksignal umgewandelt, dann misst der Zähler die Dauer einer Einzelperiode. Er ist in der Lage, die Dauer unmittelbar aufeinanderfolgender Perioden zu messen und zu speichern. Bei einer Taktfrequenz des Zählers von $200 \mathrm{MHz}$ ist die Periodendauermessung bei $1 \mathrm{MHz}$ Messfrequenz auf $+1-0.5 \%$ genau.

Ein Befehl des Rechners startet den Zählprozessor, die Messung endet nach $4000 \mathrm{Zählvorgängen,} \mathrm{wenn} \mathrm{der} \mathrm{Speicher} \mathrm{gefüllt} \mathrm{ist.} \mathrm{Die} \mathrm{Zwischen-}$ speicherung ist nötig, da der Zähler sehr viel schneller Daten liefert, als sie der Rechner einlesen kann. Aufgrund dieser Arbeitsweise des Zählprozessors lässt sich die Gesamtmesszeit T durch Wahl der Frequenzverschiebung varifieren, es ist $T=4000 / f$. Nach der Messung übernimmt der Rechner die gespeicherten Daten zur weiteren Bearbeitung.

Ein Anwendungsbeispiel

Ein typischer Anwendungsfall für die Heterodyn-Speckle-Interferometrie ist die Vermessung von Schwingungen von Lautsprechermembranen, Beispiele dafür sind in den Abbildungen 2 und 3 dargestellt $($ nächste Seite 1. Aufgetragen ist die Geschwindigkeit in Abhängigkeft von der Zeit, die Messrate betrug $400 \mathrm{kHz}$, jeder Kurvenpunkt ist der Mittelwert aus 6 Messwerten.

ADb. 2 zeigt das Verhalten eines Lautsprechers bef unterschiedichen Anregungen, bei der oberen Kurve mit Pulsen von $0.1 \mathrm{msec}$ Dauer und $1 \mathrm{kHz}$ Frequenz, bef der unteren mit Pulsen von $0.5 \mathrm{msec}$ Dauer und $200 \mathrm{~Hz}$ Frequenz.

In Abb. 3 sieht man eine sinusgrundschwingung von $200 \mathrm{~Hz}$, dabei schlägt die Membran gegen ein metallisches Hindernis, das zu Resonanzschwingungen angeregt wird und seinersefts die Membranbewegung stört. 
Abb. 2.

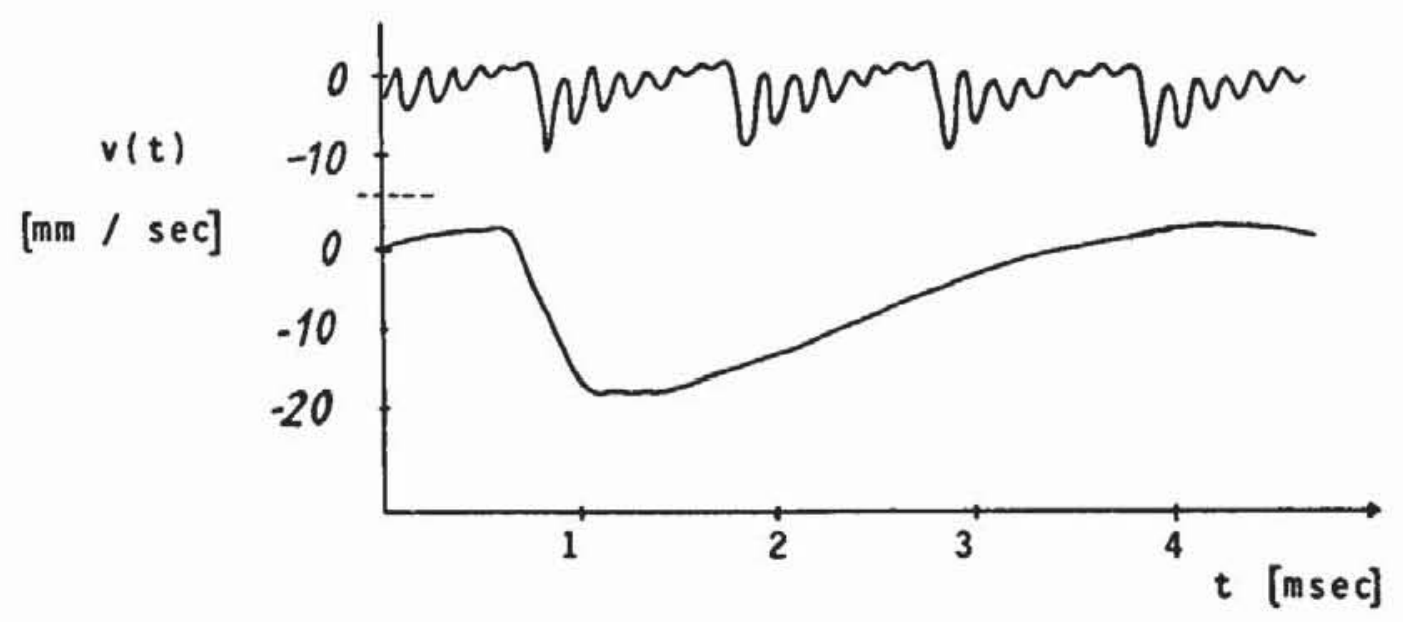

Abb. 3.

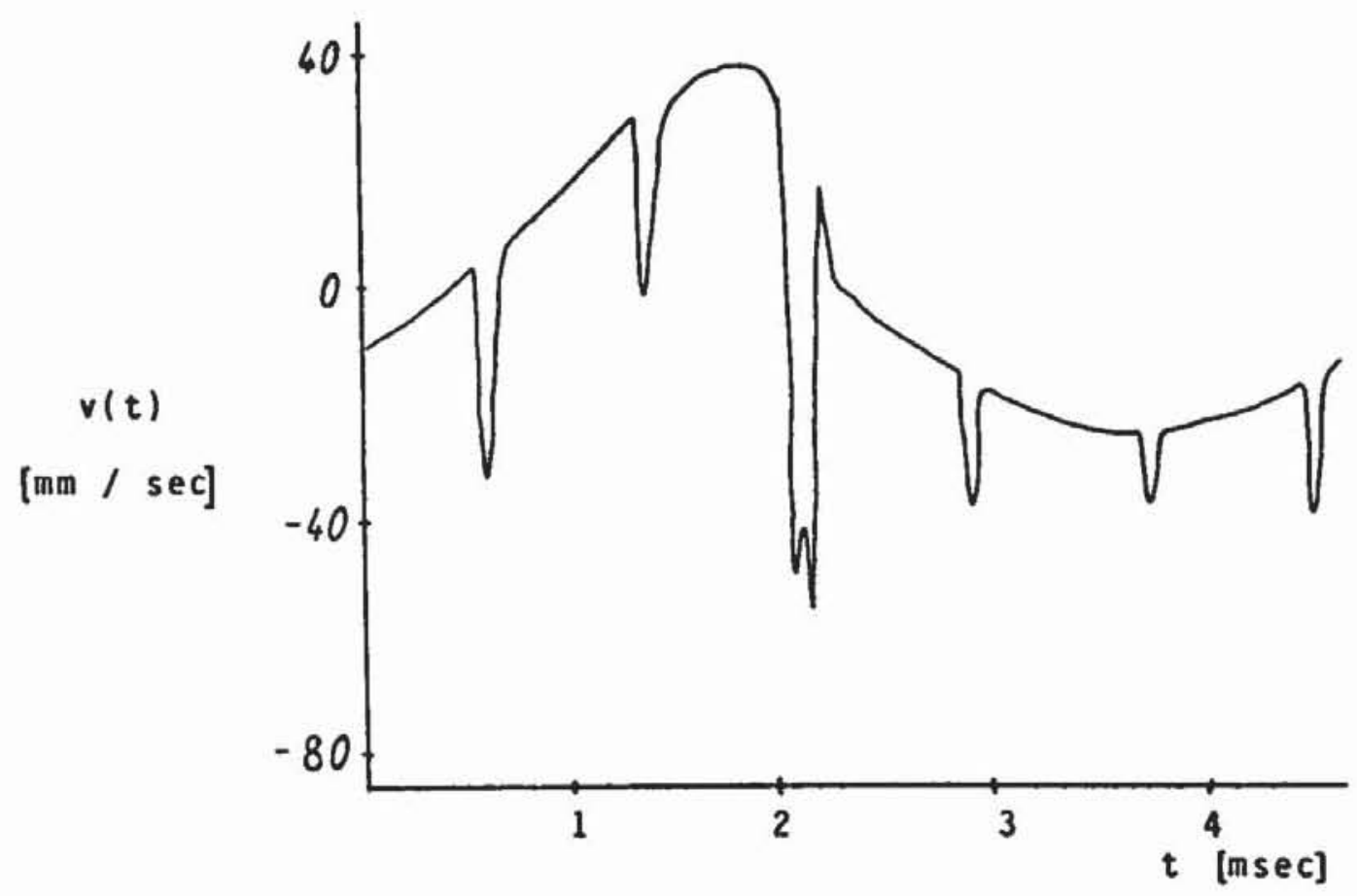

\section{Literatur}

$/ 1 / R$. Jones, C. Wykes : Holographic and Speckle Interferometry Cambridge University Press 1983

/2/ L.E. Drain: The Laser Doppler Technique Wiley \& sons, New York 1980

/3/ W.H. Stevenson: Optical Frequency Shifting by Means of a Rotating Diffraction Grating. Applied Optics $\underline{9}$ (1970), p. $649-52$

/4/ I. Peczeli, P. Richter, F. Engard: Frequency Shifted Local oscillator Produced by a Rotating Radial Grating for a Heterodyne Detector. Optica Acta $32(1985)$, p. $463-68$ 